de la lune, les planètes et les jours de la semaine qui ont connus pour l'organisme psycho-somatique comme diagramme sous le nom rHam-bcu-dbang-ldan du système de Kâlacakra. En outre les sceaux, respectivement les a misons des fantômes importants ( $T$ ib.: $1 \mathrm{Ha}$ ) de notre sphère se montrent influents comme signe de souveraineté. Ce système des images se place au service d'une connaissance pour un ordre prospère, pour un maintien et pour une activation du jeu harmonieux du dynamisme cosmique. Qu'il me soit permis de souligner que ces descriptions sont à interpréter dorénavant comme plans d'une structure cosmique.

\title{
ÜBER ÄQUIDEFORMATEN
}

\author{
J. BOLLIGER \\ 1. AEQUIDEFORMATEN \\ zu Albers flächentreuer Kegelprojektion
}

Ein Auftrag der Firma Kümmerly \& Frey zur Berechnung einer Netzprojektion für eine Karte von Europa zwischen $30^{\circ}$ und $70^{\circ}$ Breite führte mich dazu, für die gewählte «Albers flächentreue Kegelprojektion» mit den längentreuen Breitenkreisen von $45^{\circ}$ und $62^{\circ}$ die Aequideformaten zu bestimmen.

Das Längenverzerrungsverhältnis kann für jede Kartenprojektion am einfachsten durch Linien gleicher Verzerrung, Aequideformaten geriannt, dargestellt werden. Diese Linien geben das Maß und den Verlauf der Längenverzerrung für das ganze Gebiet, über das die Projektion sich ausdehnt. Für die eingangs erwähnte Albers-Projektion soll als praktisches Beispiel die Ableitung und Darstellung von Aequideformaten gezeigt werden.

Bei jeder flächentreuen Kartenprojektion ist das Verzerrungsverhältnis, wie es in Tissots Verzerrungsellipse dargestellt wird, für die beiden Ellipsenachsen reziprok. Es gilt demnach für die Maximalverzerrung $h$ in der Meridianrichtung und der senkrecht dazu verlaufenden maximalen Verzerrung $\mathrm{k}$ im Breitenkreis die Gleichung

$$
\mathrm{h} \times \mathrm{k}=1 \text { oder } \mathrm{h}=\frac{1}{\mathrm{k}}
$$

Für die beiden längentreuen Breitenkreise $45^{\circ}$ und $62^{\circ}$ ist das Verzerrungsverhältnis $\mathrm{h}=\mathrm{k}=1$. Eine Längenvergrößerung tritt ein, wenn das Verhältnis größer als 1 ist, und eine Verkleinerung, wenn es als echter Bruch unter den Wert 1 sinkt. Für unser Beispiel tritt zwischen den beiden längentreuen Breitenkreisen in der Breitenkreisrichtung eine Verkleinerung und in der Meridianrichtung eine Vergrößerung ein. Diese Verzerrung erreicht ihr Maximum bei $54^{\circ} 22^{\prime}$ mit

$$
\begin{aligned}
& \mathrm{h}=1.011 \text { in der Meridianrichtung, und } \\
& \mathrm{k}=0.989016 \text { in der Breitenkreisrichtung }
\end{aligned}
$$

Außerhalb dieser Zone nehmen die Verzerrungen mit dem Abstand von den verzerrungsfreien Breitenkreisen zu und zwar um so rascher, je größer der Abstand wird. Doch handelt es sich nun um die reziproken Werte, in der Breitenkreisrichtung wird vergrößert, in der Meridianrichtung verkleinert. Dann nimmt die Verzerrung nördlich von $62^{\circ}$ bedeutend rascher zu als südlich von $45^{\circ}$, was für die gleiche Verzerrung von 1.07 folgendes Bild zeigt:

$\begin{array}{ccc}\text { Verzerrung } & \text { Breitenkreis } & \text { Breitenabstand } \\ 1.07 & 71^{0} 07^{\prime} & 9^{0} 07^{\prime} \\ 1.00 & 62^{0} 00^{\prime} & \\ 1.00 & 45^{\circ} 00^{\prime} & 18^{\circ} 44^{\prime} \\ 1.07 & 26^{\circ} 16^{\prime} & \end{array}$


Die Aequideformaten ergeben sich aus der für diese Projektion gültigen Gleichung

$$
\mathrm{k}=\frac{\mathrm{m} \times \mathrm{n}}{\sin \delta} \quad \text { oder } \quad \mathrm{h}=\frac{\sin \delta}{\mathrm{m} \times \mathrm{n}}
$$

Dabei gelten: $\quad \mathrm{m}=$ Projektionsradius des Breitenkreises

$$
\begin{aligned}
\delta & =\text { Polabstand die es Kreises oder } \\
90^{\circ}-\delta & =\mathrm{B}, \text { geographische Breite }
\end{aligned}
$$

$\mathrm{n}$ ist für dieselbe Projektion eine Konstante, mit welcher der geographische Längenabstand vom Mittelmeridian zu multiplizieren ist, um die Meridiankonvergenz zu erhalten. Für obige Projektion ist $\mathrm{n}=0.795027$.

In der Gleichung für $k$, oder h, ist der Breitenkreisradius m eine Funktion vom Polabstand $\delta$, womit sich zeigt, daß für einen beliebigen Breitenkreis für die Breite B das Verzerrungsverhältnis kon tant ist. Demnach sind die Breitenkreise die gesuchten Aequideformaten. Setzt man in der Gleichung für $\mathrm{k}$ den Wert von $\mathrm{m}$ ein und löst nach $\delta$ auf, so führt das zu einer quadratischen Gleichung, deren Auflösung für ein gegebenes $\mathrm{k}$ die folgende Form erhält:

$$
\cos \bar{o}=\frac{\mathrm{n}}{\mathrm{k}^{2}} \pm \sqrt{1+\frac{\mathrm{n}^{2}}{\mathrm{k}^{4}}-\frac{\mathrm{n}^{2} \mathrm{~m}^{2} \mathrm{p}+2 \mathrm{n}}{\mathrm{k}^{2}}}
$$

In dieser Gleichung ist mp eine für die Projektion gegebene Konstante und bedeutet den Radius eines Kreises, der das Bild des Nordpols darstellt. Wegen dieser Abbildung des Nordpols wird die Albers Projektion auch als Kegelrumpfprojektion bezeichnet. Um mit dieser Gleichung zahlenmäßig rechnen zu können setzen wir die Werte für die Konstanten ein und erhalten:

$$
\cos \delta=\frac{0.795027}{\mathrm{k}^{2}} \pm \sqrt{1+\frac{0.632086}{\mathrm{k}^{4}}-\frac{1.624338}{\mathrm{k}^{2}}}
$$

\begin{tabular}{|c|c|c|c|c|c|}
\hline $\begin{array}{l}\text { Verzerrungs- } \\
\text { verhältnis }\end{array}$ & $\begin{array}{c}\text { Geographische } \\
\text { Breite }\end{array}$ & $\begin{array}{l}\text { Breiten- } \\
\text { intervall }\end{array}$ & $\begin{array}{l}\text { Verzerrungs- } \\
\text { verhältnis }\end{array}$ & $\begin{array}{c}\text { Geographische } \\
\text { Breite }\end{array}$ & $\begin{array}{l}\text { Breiten- } \\
\text { intervall }\end{array}$ \\
\hline 1.07 & $71^{0} 08^{\prime}$ & & 1.07 & $26^{\circ} 16^{\prime}$ & $1^{0} 58^{\prime}$ \\
\hline 1.06 & $70^{0} 25^{\prime}$ & $\begin{array}{l}43 \\
50,\end{array}$ & 1.06 & $28^{0} \quad 14^{\prime}$ & $\begin{array}{lll}1 & 58 \\
2^{0} & 06^{\prime}\end{array}$ \\
\hline 1.05 & $69^{\circ} 35^{\prime}$ & $\begin{array}{l}50 \\
57\end{array}$ & 1.05 & $30^{\circ} \quad 20^{\prime}$ & $\begin{array}{lll}2^{0} & 16^{\prime}\end{array}$ \\
\hline 1.04 & $68^{\circ} 38^{\prime}$ & $1^{0} 07$, & 1.04 & $32^{\circ} 36^{\prime}$ & $2^{0} 29^{\prime}$ \\
\hline 1.03 & $67^{\circ}, 31^{\prime}$ & $1^{0} \quad 21^{\prime}$ & 1.03 & $35^{0} 05^{\prime}$ & $2^{0} \quad 46^{\prime}$ \\
\hline 1.02 & $66^{\circ} 10^{\prime}$ & $1^{0} 44^{\prime}$ & 1.02 & $37^{0} 51^{\prime}$ & $3^{0} 111^{\prime}$ \\
\hline 1.01 & $64^{\circ} 26^{\prime}$ & $\begin{array}{ll}1 & 44 \\
2^{0} & 26^{\prime}\end{array}$ & 1.01 & $\begin{array}{ll}41^{\circ} & 02 \\
\end{array}$ & $3^{0} 58^{\prime}$ \\
\hline 1.00 & $62^{0} 00^{\prime}$ & $\begin{array}{ll}5^{0} & 10^{\prime}\end{array}$ & 1.00 & $\begin{array}{ll}45^{\circ} & 00^{\prime}\end{array}$ & $6^{0} \quad 45^{\prime}$ \\
\hline 0.99 & $56^{0} \quad 50^{\prime}$ & & 0.99 & $51^{0} 45^{\prime}$ & \\
\hline
\end{tabular}

Das Doppelvorzeichen der Wurzel führt zu zwei Lösungen für ein gegebenes $k$, denen zwei Breitenkreise mit derselben Verzerrung als Aequideformaten entsprechen. Wählen wir für $k$ ein Intervall von 0.01 , was $1 \%$ Längenverzerrung entspricht, so erhalten wir die folgende Zusammenstellung der Aequideformaten:

Diese Zahlen geben ein anschauliches Bild über die Größen und Änderungen der Längenverzerrung im Gebiet der Projektion. Am günstigsten liegt die Zone zwischen den längentreuen Breitenkreisen von $45^{\circ}$ und $62^{\circ}$, wo auf ein Verzerrungsintervall von 0.01 eine Zonenbreite von $5^{\circ} 10^{\prime}$ nördlich, und von $6^{\circ} 45^{\prime}$ südlich kommt. Das Maximum der Längenverzerrung mit 0.989 tritt bei $54^{\circ} 22^{\prime}$ Breite auf in dieser Zone. Von Bedeutung ist auch die Feststellung, daß nördlich vom 62. Breitengrad die Verzerrungszunahme im Mittel doppelt so rasch erfolgt als südlich des 45. Breitengrades, so daß die südlichen Gebiete bedeutend günstiger wegkommen in der Längenverzerrung.

Über die Verzerrungsgrößen für einzelne Gebiete soll die folgende Kartenskizze orientieren. 


\section{AEQUIDEFORMATEN DER FLÄCHENVERZERRUNG} bei der winkeltreuen schiefachsigen Zylinderprojektion der Schweiz

Wenn für eine Landesvermessung, die auch der Grundbuchvermessung und damit der genauen Arealbestimmung aus Plänen dient, eine winkeltreue Projektion gefordert wird, so ist daran die weitere Bedingung zu knüpfen, daß ihre Flächenverzerrung möglichst klein sei. Dies kann durch eine günstige Lage der Projektionsfläche erreicht werden.

Da die flächentreue Bonne-Projektion, die der Landesvermessung für die Dufour- und Siegfriedkarte zugrunde liegt, mit ihren großen Winkelverzerrungen für die neuen Triangulationen einzelner Kantone, die anschließend an das Erscheinen der Dufourkarte zur Erneuerung ihres Katasterwesens erfolgten, nicht mehr genügte, führte dies zur Einführung von behelfsmäßigen Koordinatensystemen. So wurde die Schweiz gegen das Ende des letzten Jahrhunderts schließlich in 19 verschiedene kantonale Koordinatensysteme aufgeteilt.

Im Jahr 1883 begann auf Anregung der Landestopographie die Schweizerische geodätische Kommission mit Studien zur Wahl eines für das ganze Land verwendbaren winkeltreuen Projektionssystems. Doch erst um die Jahrhundertwende, als die Landestopographie mit den Studien für neue Landeskarten begann, hat in ihrem Auftrag Ingenieur M. Rosenmund ein winkeltreues Projektionssystem entwickelt, das im Jahr 1903 angenommen wurde und seitdem allen Vermessungsarbeiten unseres Landes als Grundlage dient.

Diese neue Übertragungsart vom Referenz-Ellipsoid in die Projektionsebene ist eine Doppelprojektion. Zuerst wird vom Ellipsoid nach den vom deutschen Mathematiker C. F. Gauß entwickelten Projektionsgesetzen winkeltreu auf eine Kugel übertragen. Dann folgt von dieser Kugel die winkeltreue Übertragung auf einen schief zur Erdachse liegenden Zylinder, dessen Berührungskreis als Großkreis durch das Projektionszentrum Bern geht und senkrecht zum Berner Meridian liegt.

Die Längen- und Flächenverzerrungen bei der Gauß-Projektion sind so klein, daß hier nicht weiter darauf eingetreten werden soll. Anders ist es bei der Projektion von der Kugel auf den $Z$ ylinder, handelt es sich dabei doch um die als Merkator-Projektion bekannte winkeltreue Zylinderprojektion, die im 16. Jahrhundert von G. Kremer ersonnen wurde. Bei dieser ist der Aequator der Berührungskreis mit dem Zylinder, der längentreu abgebildet und in der Zylinderabwicklung zur Geraden wird. Die Breitenkreise sind parallel zum Aequator liegende Grade, die im Verhältnis von 1/ cos B vergrößert werden. Die ebenfalls als Gerade abgebildeten Meridiane unterliegen derselben nur von der Breite B abhängigen Vergrößerung.

Dieselben Verzerrungsgesetze gelten auch für un:ern schiefachsig in Bern anliegenden Zylinder, nur tritt an Stelle des Abstandes B vom Aequator der Abstand X von der längentreu abgebildeten Y-Achse. Die Merkatorprojektion ist wegen ihrer starken Längen- und Flächenverzerrung nicht allgemein verwendbar, wenn sie auch für Schifffahrtzwecke ihre Vorteile hat. Schon bei $60^{\circ}$ Breite tritt eine vierfache Flächenvergrößerung auf und das Bild der Erdpole fällt ins Unendliche. Für die kleine Nord-SüdAusdehnung der Schweiz ist die Flächenvergrößerung tragbar und fällt nicht aus dem Rahmen der bei allen winkeltreuen Abbildungen eintretenden Verzerrung.

Die Forderung der Winkeltreue ist für eine Projektion erfüllt, wenn die beiden Halbachsen von Tissots Verzerrungsellipse gleich groß werden, also $h=k$ ist, womit die Verzerrungsellipse zum Kreis wird. Zur Berechnung der Flächenverzerrung für die winkeltreue schiefachsige $Z$ ylinderprojektion der Schweiz gehen wir von der folgenden Gleichung aus, welche die beiden ersten Glieder einer Reihenentwicklung darstellt. Darin bedeuten $d$ s' ein Linienelement in der Projektionsebene und d s dasselbe Element auf der Kugel, deren Radius mit R bezeichnet ist. Die Quadrate dieser beiden ergeben Flächenelemente.

$$
\frac{\mathrm{d} s^{2}}{\mathrm{~d} \mathrm{~s}^{2}}=1-\frac{\mathrm{X}^{2}}{\mathrm{R}^{2}}+\ldots
$$

Aus die:er Gleichung geht hervor, wie schon erwähnt, daß das Verzerrungsverhältnis nur von der Größe von $X$ abhängt und bei deren gegebener Größe parallel zur Y- 


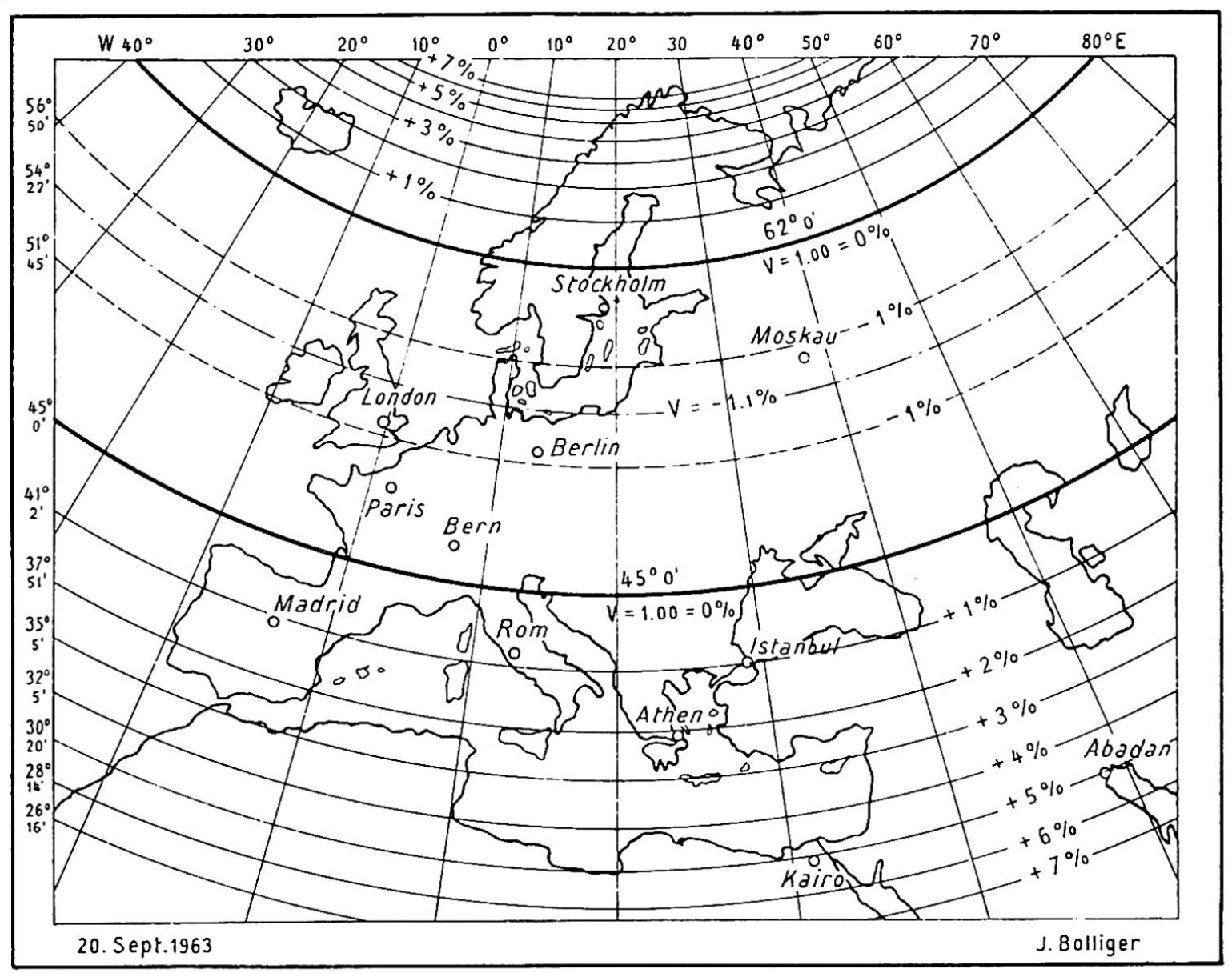

Figur 1: Äquideformaten zu Albers flächentreuer Kegelprojektion

Achse konstant verläuft, was einer Aequideformate entspricht. Wird die Flächenverzerrung in Promillen p ausgedrückt, so führt eine Umwandlung obiger Gleichung zur Form:

$$
\mathrm{p}=\frac{1000}{\mathrm{R}^{2}} \times \mathrm{X}^{2}
$$

Setzen wir den Kugelradius $\mathrm{R}=6378.8 \mathrm{~km}$ in diese Gleichung ein und nehmen auch die Größe X in Kilometern, so gibt das die folgende Rechenformel :

$$
\mathrm{p}=2.458 \times \mathrm{X}^{2} \times 10^{-5}
$$

Oder bei gegebenem p zur Zeichnung von Aequideformaten im Intervall z. B. von $0.02 \%$, lösen wir obige Gleichung nach $X$ auf und erhalten wegen des doppelten Vorzeichens der Wurzel die beiden symmetrisch zur Y-Achse liegenden Werte von $\mathrm{X}$ in Kilometereinheiten:

$$
\mathrm{X}= \pm 201.7 \times \sqrt{\mathrm{p}}
$$

Die folgende Aufstellung zeigt für die im Intervall von $0.02 \%$ zunehmende Flächenverzerrung den zugehörenden X-Abstand von der Y-Achse. In der dritten Kolonne ist dem stetigen Intervall von $\mathrm{p}$ das zugehörende Intervall von $\mathrm{X}$ beigefügt, das zeigt, wie die Zunahme von $\mathrm{X}$ immer kleiner wird, je größer der Wert von $X$ ist. Der Abstand der Aequideformaten wird also verkleinert, je weiter die Orte von der Y-Achse entfernt liegen, d.h. um so rascher nimmt die Flächenverzerrung zu. 


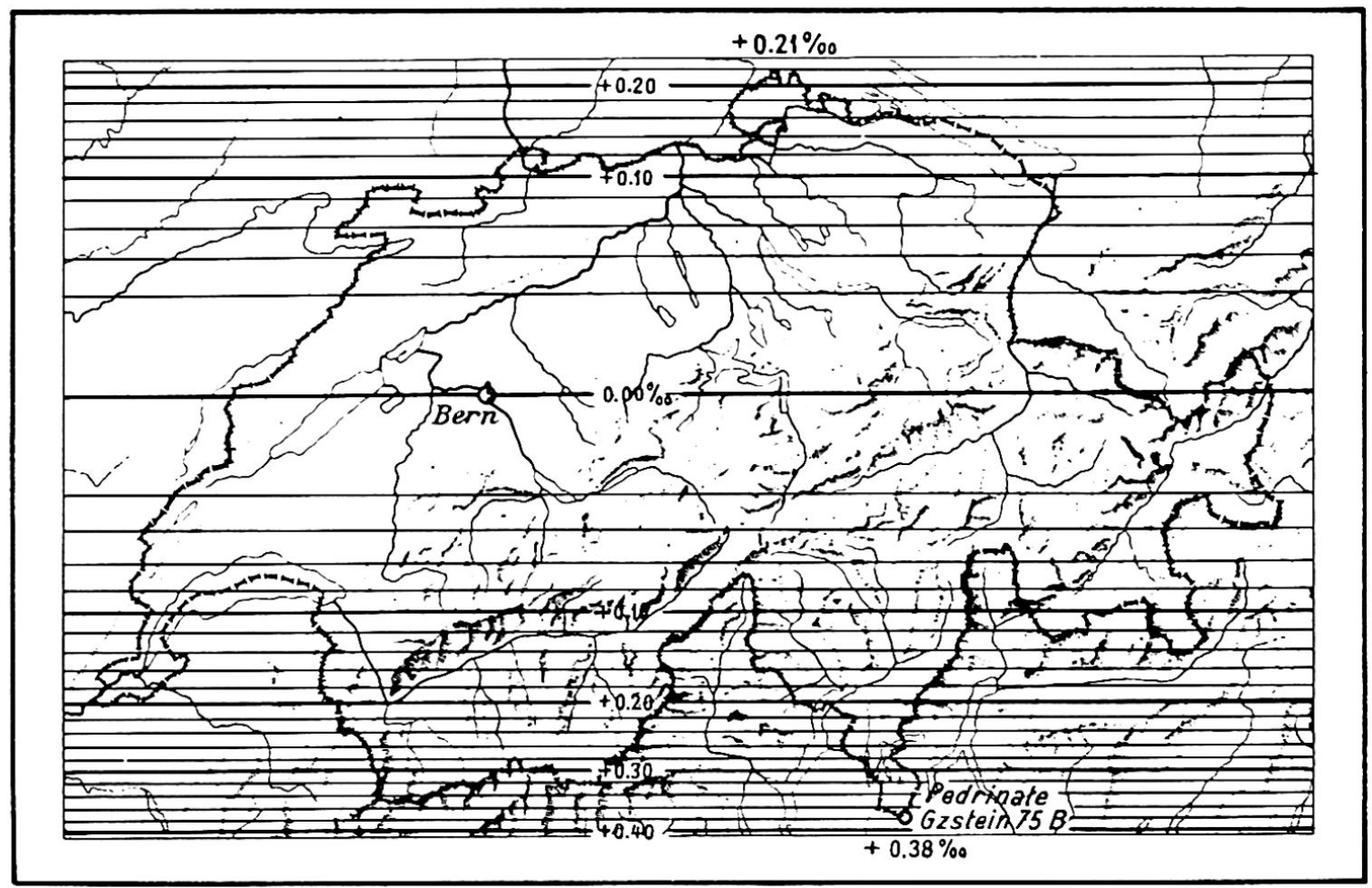

Figur 2: Äquideformaten der Flächenverzerrung bei der winkeltreuen schiefachsigen Zylinderprojektion der Schweiz

\begin{tabular}{|c|c|c|c|c|c|}
\hline $\begin{array}{c}\mathrm{p} \\
\%\end{array}$ & $\begin{array}{c} \pm \mathrm{X} \\
\mathrm{km}\end{array}$ & $\begin{array}{c}\text { Differenz } \mathrm{X} \\
\text { in } \mathrm{km}\end{array}$ & $\begin{array}{c}\mathrm{p} \\
\mathrm{\%} / 00\end{array}$ & $\begin{array}{c} \pm \mathrm{X} \\
\mathrm{km}\end{array}$ & $\begin{array}{c}\text { Differenz } \mathrm{X} \\
\text { in } \mathrm{km}\end{array}$ \\
\hline & & & & & \\
0.00 & 0.0 & 28.5 & 0.20 & 90.2 & 4.4 \\
0.02 & 28.5 & 11.8 & 0.22 & 94.6 & 4.2 \\
0.04 & 40.3 & 9.1 & 0.24 & 98.8 & 4.0 \\
0.06 & 49.4 & 7.7 & 0.26 & 102.8 & 3.9 \\
0.08 & 57.1 & 6.7 & 0.28 & 106.7 & 3.8 \\
0.10 & 63.8 & 6.1 & 0.30 & 110.5 & 3.6 \\
0.12 & 69.9 & 5.7 & 0.32 & 114.1 & 3.5 \\
0.14 & 75.6 & 5.1 & 0.34 & 117.6 & 3.4 \\
0.16 & 80.7 & 4.9 & 0.36 & 121.0 & 3.4 \\
0.18 & 85.6 & 4.6 & 0.38 & 124.4 & 3.2 \\
0.20 & 90.2 & & 0.40 & 127.6 & \\
\hline
\end{tabular}

Aus diesen Werten läßt sich für eine beliebige Größe von $X$ die Flächenverzerrung abschätzen oder, genauer, interpolieren. Anschaulicher orientiert über die Verzerrungsverteilung das folgende Kärtchen der Aequideformaten der Flächenverzerrung. Deutlich erkennt man, wie in einer breiten Mittelzone bei der Y-Achse die Verzerrung nur langsam zunimmt, um dann bei gröBerer Entfernung um so rascher zu wachsen. Die größte Flächenverzerrung haben wir im Norden bei Bargen mit ${ }^{0} .21 \%$ und im Süden bei Chiasso mit ${ }_{0} 0.38 \%$.

Nehmen wir bei Schaffhausen ein Gemeindeareal von $1 \mathrm{~km}^{2}$ an mit der Flächenverzerrung von $0.18 \%$, so gibt die Projektionsverzerrung den Betrag von $180 \mathrm{~m}^{2}$, um den das aus Grundbuchplänen auf den $\mathrm{m}^{2}$ bestimmte Areal zu groß ist. Die Fläche des Kantons Zürich mit $1729 \mathrm{~km}^{2}$ erleidet eine Projektionsvergrößerung von 22 ha und die ganze Schweiz mit $41295 \mathrm{~km}^{2}$ eine solche von $2.6 \mathrm{~km}^{2}$.

Hierzu muß festgehalten werden, daß der Projektionsvergrößerung eine Verkleinerung gegenüberwirkt, weil unsere Vermessungsgrundlagen auf den Meereshorizont re- 


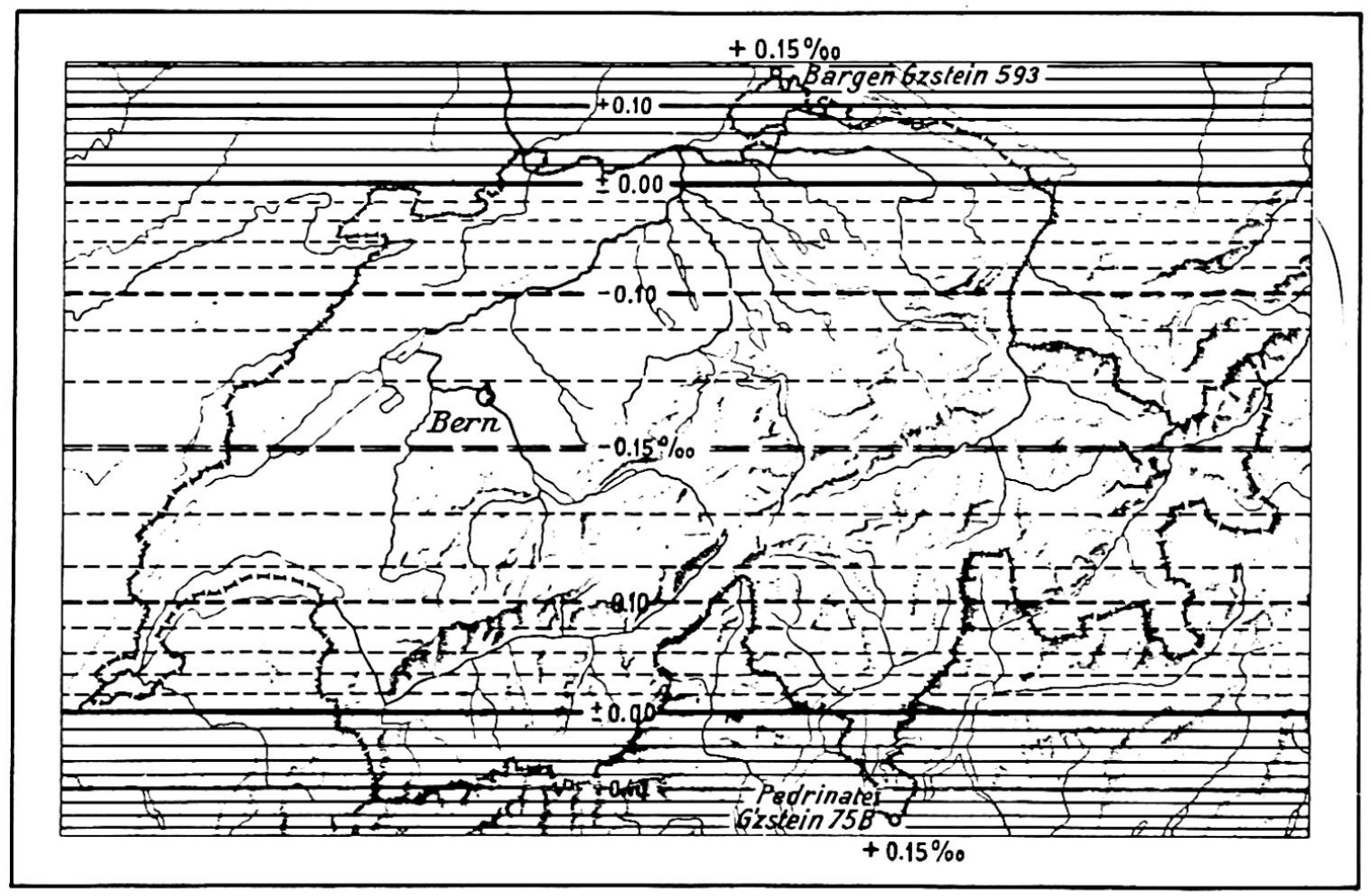

Figur 3: Äquideformaten der Flächenverzerrung bei winkeltreuer Projektion auf einen Schnittzylinder mit maximaler Flächenverzerrung von $\pm 0.15 \%$

duziert sind. Das Zusammenwirken dieser beiden Verzerrungen gibt die Korrektur, die an der Arealbestimmung anzubringen ist, welche aus Grundbuchplänen erfolgt.

Es wurde eingangs geschrieben, daß die Größe der Projektionsverzerrung durch die Lage der Projektionsfläche beeinflußt werden kann. Würden wir den Berührungskreis des Zylinders um $14.4 \mathrm{~km}$ nach Süden verschieben, so hätten wir für den nördlichsten und südlichsten Punkt der Schweiz die gleiche Flächenverzerrung von $0.30 \%$.

Auch die:e Extremalverzerrungen lassen sich noch auf die Hälfte verkleinern, wenn statt dem Berührungszylinder ein Schnittzylinder gewählt wird. Für die beiden verzerrungsfreien Schnittkreise wäre dann im Norden $X=+63.3 \mathrm{~km}$ und im Süden $X=$ $-92.4 \mathrm{~km}$. Zwischen diesen beiden Kreisen tritt nun eine Verkleinerung ein, die bei $X$ $=-14.4 \mathrm{~km}$ ihr Maximum mit $-0.15 \%$ orreicht. Die beiden andern Maximalverzerrungen haben wir am nördlichsten und südlichsten Punkt der Schweiz mit $+0.15 \%$. Das folgende Kärtchen orientiert nun über die Lage der Aequideformaten bei diesem Schnittzylinder.

\section{DIE WINKELVERZERRUNGEN DER BONNE-PROJEKTION}

Für die flächentreue Bonne-Projektion der Dufour- und der Siegfriedkarte, die wegen ihrer großen Winkelverzerrungen im Jahre 1903 durch die winkeltreue schiefachsige Zylinderprojektion in unserer Landesvermessung er.etzt wurde, soll im folgenden auf diese Winkelverzerrung eingetreten werden. Ohne auf die Ableitung der Gleichung für die Winkelverzerrung hier näher eintreten zu können, führen wir diese wie folgt an:

$$
\triangle a=\sin ^{2} a\left[\mathrm{Y} \times \mathrm{X}+\operatorname{tg} b_{0}\left(\mathrm{Y} \times \mathrm{X}^{2}-1 / 2 \mathrm{Y}^{3}\right)+\ldots\right]
$$

In dieser Gleichung ist $\alpha$ der Azimutwinkel, $\triangle a$ seine Verzerrung, $Y$ und $X$ sind die Koordinaten des Ortes, an welchem die Azimutmessung erfolgt, wobei mit genügender Genauigkeit die Zylinderkoordination verwendet werden können. Die Konstante tg bo bezieht sich auf die geographische Breite bo des Projektionszentrums Bern. Die 


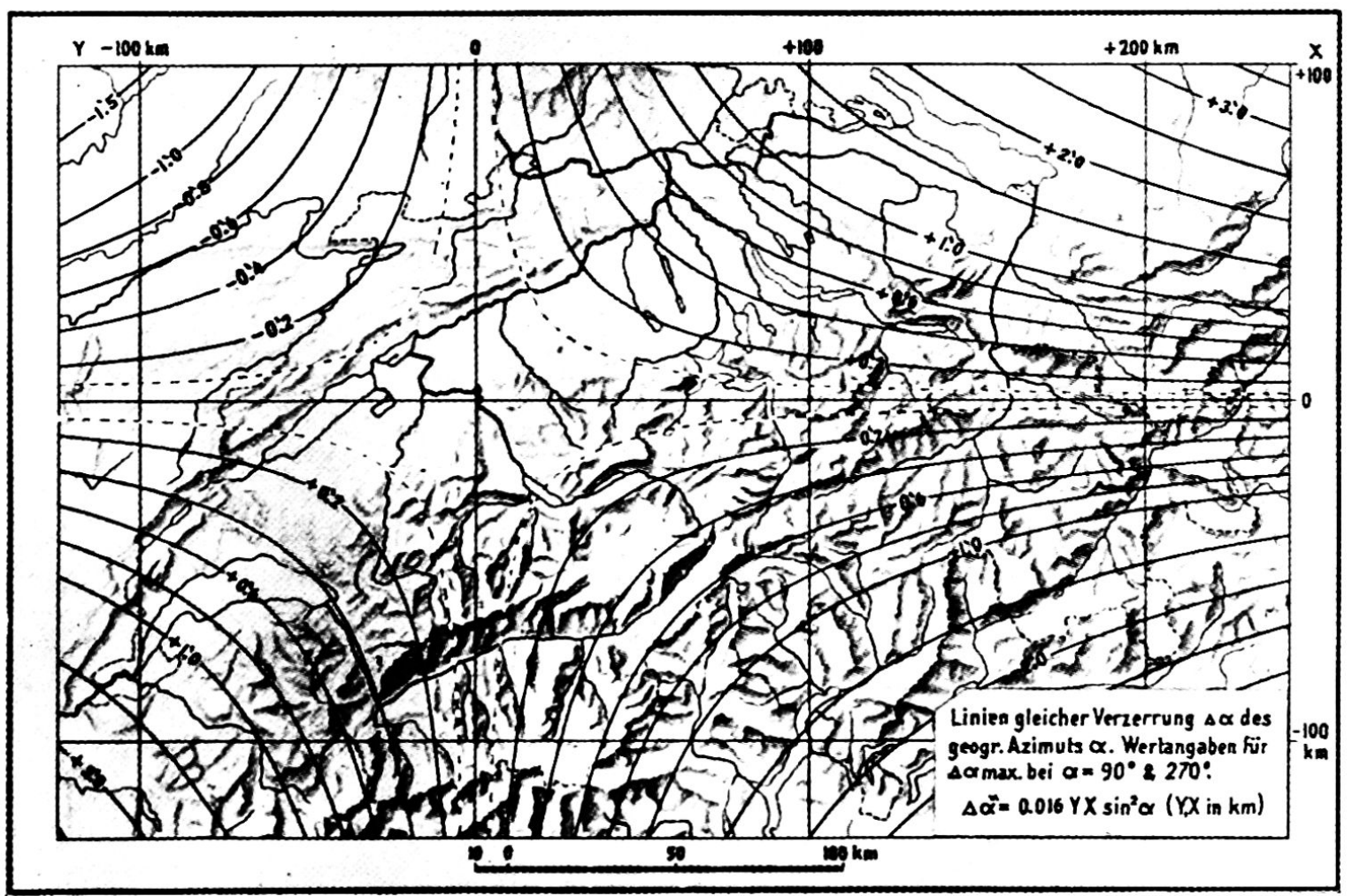

Figur 4: Äquideformaten der Bonne-Projektion für die maximalen Winkelverzerrungen des Azimuts

Glieder mit tg bo können wir vernachläßigen und gelangen zur folgenden vereinfachten Gleichung für die Verzerrung $\triangle a$ des Azimutwinkels $a$

$$
\triangle a=\sin ^{2} a \times \mathrm{Y} \times \mathrm{X}
$$

Wir sehen aus dieser Gleichung, daß die Winkelverzerrung $\triangle \alpha$ von der Größe des Azimutwinkels $\alpha$ abhängt und für $\alpha=0^{\circ}$ und $180^{\circ}$ zu Null wird. Ihr Maximum erreicht sie für $\sin \alpha= \pm 1$, d.h. für $\alpha=90^{\circ}$ und $270^{\circ}$. Die Winkelverzerrung ist aber auch von der Ortslage abhängig, sie ist in den beiden Koordinatenachsen gleich Null und nimmt mit zunehmendem $\mathrm{Ab}$ tand von diesen zu. Wegen der Vorzeichen der Koordinaten ist die Verzerrung im 1 . und 3. Achsenquadranten positiv, also eine Vergrößerung, und im 2. und 4. Quadranten negativ, eine Verkleinerung.

Um die obige analytische Gleichung zum Rechnen verwenden zu können, müssen wir rechts mit $Q$ multiplizieren und durch $\mathrm{R}^{2}(\mathrm{R}=$ Kugelradius $)$ dividieren, sie lautet nun:

$$
\triangle \iota^{\prime \prime}=\frac{\varrho^{\prime \prime}}{\mathrm{R}^{2}} \times \sin ^{2} \iota \times \mathrm{Y} \times \mathrm{X}
$$

Setzen wir den Kugelradius $\mathrm{R}=6378.8 \mathrm{~km}$ ein und nehmen die Koordinaten $\mathrm{X}$ und $\mathrm{Y}$ in Kilometereinheiten, so gibt das die

\section{Rechenformel zum Bestimmen der Verzerrung des Azimutwinkels \\ $\triangle \imath^{\prime \prime}=0.005069 \times \sin ^{2} \iota \times \mathrm{Y} \times \mathrm{X}$ für alte Winkelteilung \\ $\triangle c^{\prime \prime}=0.015646 \times \sin ^{2} a \times \mathrm{Y} \times \mathrm{X}$ für neue Winkelteilung}

Für den Ort der größten Winkelverzerrung, in Campocologno im Puschlav, erhalten wir mit obiger Rechenformel eine maximale Winkelverzerrung von 262" in never, oder $85^{\prime \prime}$ in alter Teilung. Die obige Karte der Aequideformaten orientiert nun über die maximalen Verzerrungen des Azimutwinkels für einen beliebigen Ort der Schweiz. 
Für die Zeichnung von Aequideformaten mit maximaler Winkelverzerrung ist $\sin ^{2} \alpha$ $=1$. Damit wird die obige Verzerrungsformel zur Gleichung einer Hyperbel, bezogen auf das Koordinatenachsenkreuz als Asymptoten, wie es aus dem Kärtchen hervorgeht.

$\mathrm{Da}$ bei derart großen, in die Minuten gehenden Verzerrungen des Azimutwinkels, die nicht nur von der Ortslage, sondern auch von der Größe des Azimutwinkels abhängen, die Bonne-Projektion verlassen und eine winkeltreue Projektion eingeführt wurde, erscheint nun selbstverständlich. Bei der geforderten Genauigkeit der Winkelmessung sind derartige Verzerrungskorrekturen nicht mehr tragbar. Wurde doch früher für die Triangulation 4. Ordnung zwischen zwei unabhängigen Winkelmessungen eine Übereinstimmung von mindesten; 11 " gefordert, wofür heute ein höchstzuläßiger mittlerer Winkelfehler von nur \pm 4 " gestattet ist.

Auch bei einer winkeltreuen Projektion treten Winkelverzerrungen auf, da die Winkeltreue nur im unendlich Kleinen gilt, praktisch jedoch für kleine Richtungsdistanzen keine spürbaren Verzerrungen gibt. Diese Verzerrungen, die mit der Azimutreduktion bei der Triangulation höherer Ordnung korrigiert werden, sind bei der Triangulation 4. Ordnung nicht mehr spürbar.

\section{LIGNES D'ÉGALE DÉFORMATION}

La déformation des longueurs dans la «projection conique équivalente d'Albers» est illustrée par un exemple s'étendant sur tout la surface d'une carte d'Europe. La déformation maximale des longueurs par cette projection est constante sur un parallèle. Les parallèles sont ainsi des lignes d'égale déformation. Entre les deux parallèles de longueur conservée de $45^{\circ}$ et $62^{\circ}$ représentée ici, on constate un raccourcissement des longueurs, en dehors d'eux un allongement. A un éloignement croissant les deux parallèles de longueur conservée correspond une déformation croissante. Perpendiculairement aux parallèles, il y a dans la direction du méridien une seconde déformation maximale, complémentaire de la déformation du parallèle.

Un second exemple pour la «projection cylindrique conforme» des mensurations fédérales traite de la déformation des surfaces. Ici les lignes d'égale déformation sont parallèles au cercle de contact du cylindre passant par Berne, parallèles donc à l'axe des Y de la projection. La déformation des surfaces est un agrandissement d'autant plus rapide que croit l'éloignement de l'axe des Y, comme le montre le croquis de toute la Suisse.

Les valeurs extrèmes de $0,21 \% 0$ au nord et de $0,36 \% 0$ au sud peuvent être amenées à une même valeur de $0.30 \%$ en déplacant le cercle de contact du cylindre. En choisissant un cylindre seccant au lieu d'un cylindre de contact, les déformations extrèmes des surfaces peuvent être réduites de moitié, pour les $\mathrm{X}=-14,4 \mathrm{~km} B-0,15 \%$ et pour les extrèmes nord et sud de la Suisse à

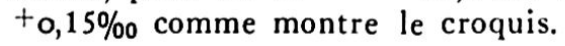

Un troisième exemple intéressant la déformation de l'angle est montré dans la déformation de l'angle azimutal dans la «projection de Bonne». Cette déformation atteint son maximum pour l'azimut de $90^{\circ}$ et de $270^{\circ}$, elle est nulle pour $0^{\circ}$ et $180^{\circ}$. Les lignes d'égale déformation sont des hyperboles, centrées sur la croisée des axes de la projection. La déformation augmente avec l'éloignement des axes, un agrandissement dans le 1 er et le $3 \mathrm{e}$ quadrant et une réduction dans le $2 \mathrm{e}$ et $4 \mathrm{e}$ quadrant comme le montre notre croquis.

\section{JAHRESVERSAMMLUNG \\ DER SCHWEIZERISCHEN NATURFORSCHENDEN GESELLSCHAFT IN ZÜRICH}

Die 144. Jahresversammlung der SNG findet vom Freitag, den 9. bis Sonntag, den 11. Oktober 1964 in Zürich statt. Das erste Einladungszirkular wird am 10. Mai versandt. Die Kollegen werden freundlich eingeladen, sich mit Beiträgen an der Sitzung für Geographie und Kartograpie zu beteiligen. Anmeldungen hierzu sind bis spätestens 31. Mai an den Präsidenten des VSGG, Professor Dr. E. Paillard, Lausanne, Avenue Jomini 1, erbeten. 